\title{
Implementation of experimental design methodology in preparation and characterization of zolmitriptan loaded chitosan nanoparticles
}

\author{
*Satish K. Mandlik ${ }^{1,2}$ and Nisharani S. Ranpise ${ }^{1}$ \\ ${ }^{1}$ Department of Pharmaceutics, Sinhgad College of Pharmacy, Pune, Maharashtra, India \\ ${ }^{2} J a w a h a r l a l$ Nehru Technological University, Hyderabad, Telangana, India
}

\begin{abstract}
The present study investigated the implementation of $3^{2}$ factorial design of experiment and statistical analysis for the optimization of chitosan nanoparticles containing zolmitriptan an antimigraine drug. The influence of chitosan concentration $\left(\mathrm{X}_{1}\right)$ and sodium tripoly phosphate $\left(\mathrm{X}_{2}\right)$ on responses namely nanoparticle size $\left(\mathrm{Y}_{1}\right)$, and entrapment efficiency $\left(\mathrm{Y}_{2}\right)$, was studied. As per design, nine runs of nanoparticles were prepared by modified ionic gelation method using high speed vortex mixing. The particle size was found in the range of 151-880 nm and entrapment efficiency was 72.3-81.2\%. A statistical analysis was performed using licensed design expert software V.8.0 with respect to ANOVA, regression analysis. The contour plots and response surface plots showed visual representation of relationship between the experimental responses and the set of independent variables. Regression model equations were validated by a numerical and graphical optimization method. Further, optimized drug loaded nanoparticles showed $+23.7 \mathrm{mV}$ zeta potential indicating storage stability, electron micrograph reflects spherical shape and mixed type of drug release followed by Fickian diffusion $(n=0.266)$ was observed. Thus, using systematic factorial design approach, desirable goals can be achieved in shortest possible time with lesser number of experiments which was proven to be an effective tool in quality by design.
\end{abstract}

Key Words: Factorial design; regression; nanoparticles; chitosan; zolmitriptan.

\section{INTRODUCTION}

Experimental design methodology or design of experiments (DOE) has been proven a powerful tool in research and development and is used to correlate the relationship between independent variables (factors) i.e. critical to process (CTPs) and dependent variables (responses) i.e. critical to quality (CTQs). DOE is an important and systematic tool used in quality by design system and implemented to draw statistical interpretations with minimum number of experiments to estimate design space (Antony, 2014; Zhang and Mao, 2016). Different types of designs are used in pharmaceutical research and development for optimization of formulations with less runs, traces problems and statistically desired solution. A factorial design is used to understand the effect of two or more independent variables upon dependent variables and suitable for understanding the interaction of variables (Lewis et al., 1998; Neves et al., 2014).

Chitosan is a polysaccharide, biodegradable, biocompatible, cationic polymer comprises of copolymers of glucosamine and $\mathrm{N}$-acetyl glucosamine linked by $\beta-(1-4)$ linkages. Chitosan obtained by partial deacetylation of chitin obtained from crustacean sea animals. Its versatility and abundance render its wide application in pharmaceutical, cosmeceutical and biomedical engineering. It is commercially available in various grades with different molecular weights and degrees of deacetylation (Amidi et al., 2010; Mincea et al., 2012; Tiyaboonchai, 2003).

Chitosan nanoparticles are most commonly used in targeted drug delivery due to its biodegradability and advantageous over lipidic nanoparticles with respect to its
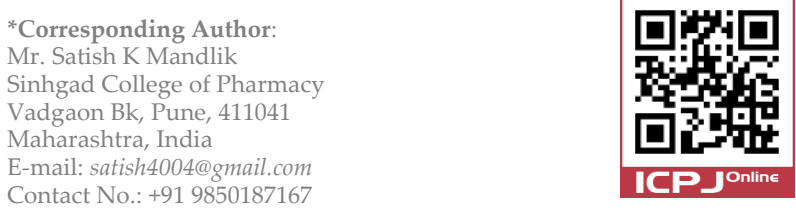

long shelf life and maximum drug entrapment (Agnihotri et al., 2004; Christoper et al., 2014).

Zolmitriptan (ZMT) is a second-generation triptan derivatives, selective serotonin 5-HT receptor agonist used in all types of migraine patients. (Charlesworth and Dowson., 2002). Patients with migraine generally suffer from nausea and vomiting; oral dosage forms can therefore be inconvenient or unacceptable (Uemura et al., 2005). An intranasal administration of ZMT offers advantages to patients, particularly those seeking a more rapid onset of effect (Goadsby and Yates, 2006). The rapid clearance of the drug from the nasal cavity and hepatic metabolism could lead to only $40 \%$ absolute bioavailability by nasal and oral administration. The ZMT has short half-life of 2.5-3 h (Dixon and Warrander, 1997).

The objective of the present study relates to development and evaluation of chitosan nanocarriers of ZMT using $3^{2}$ factorial design of experiments as a quality by design tool. The study reveals the effect of independent variables namely concentration of chitosan and sodium tripoly phosphate (STPP) cross linker on nanoparticle size and entrapment efficiency.

\section{MATERIALS AND METHODS}

\section{Materials}

ZMT was obtained from Cipla Ltd, Mumbai, India, chitosan (viscosity $35 \mathrm{cps}$, deacetylation degree 95\%) was purchased from Loba Chemie, Mumbai, India. STPP, glacial acetic acid, potassium dihydrogen phosphate, sodium hydroxide and all other chemicals were of analytical grade, purchased from Loba Chemie, Mumbai, India.

\section{Drug-polymer interaction studies}

The differential scanning calorimetry (DSC) thermograms of pure ZMT and physical mixtures of the drug with excipients were recorded by using a DSC (DSC 823 Mettler Toledo, Japan) equipped with the computerized data station. The same mixtures were also analyzed for IR spectra 
analysis using FTIR spectrophotometer (Perkin Elmer, Spectrum BX, USA) over the frequency range of 4000-500 $\mathrm{cm}^{-1}$.

\section{Preparation of ZMT loaded Nanoparticles}

Chitosan nanoparticles were prepared by ionic gelation of chitosan with STPP (Koukaras et al., 2012) (Wang et al., 2008). Different concentrations of chitosan $(1-3 \mathrm{mg} / \mathrm{ml})$ were dissolved in $1 \% \mathrm{v} / \mathrm{v}$ aqueous acetic solution and ZMT was dissolved in the solutions. The ZMT-chitosan solution was vortexed by using a cyclomixer (Cyclo CM101, Remi Equipments, Mumbai, India). The STPP solution (0.5-2 $\mathrm{mg} / \mathrm{ml}$ ) was gently added to the ZMT-chitosan solution during vortexing to form nanoparticles further these are freeze dried (Christ Alpha 1-4 LD, Germany) (Mandlik et al., 2013).

\section{Factorial Design of Experiments}

A two factor, three level factorial design was implemented to study the individual effects of each factor and their interaction on the characteristics of nanoparticles (Parhi et al., 2015; Mehta et al., 2007). The independent variables, $X_{1}$ chitosan and $X_{2}$ STPP concentrations were set at three levels to obtain nine possible runs. The critical quality attributes of nanoparticles were $\mathrm{Y}_{1}$ particle size (PS) and $\mathrm{Y}_{2}$ entrapment efficiency (EE) and thus were selected as the response variables. A $3^{2}$ factorial design design matrix is summerized in Table 1 and 2.

\section{Particle size analysis}

The particle size analysis of all nine runs was measured using laser diffraction technique (Helos, Sympatec $\mathrm{GmbH}$, Germany). All measurements were performed in triplicate.

\section{Entrapment efficiency (EE)}

The amount of ZMT entrapped in chitosan nanoparticles was determined by indirect method (Wang et al., 2008). An aliquot of the supernatant containing the free drug was collected immediately after ultracentrifugation (412LAC, Remi Equipments, Mumbai, India) of nanosuspension and analyzed by UV spectrophotometry at $222 \mathrm{~nm}$. All measurements were performed in triplicate. The entrapment efficiency of ZMT was calculated according to Equation 1.

$E E(\%)=\frac{\text { Total amount of } Z M T-\text { Amount of free } Z M T}{\text { Total amount of } Z M T} \times 100 \ldots \ldots$ (1)

\section{In-vitro drug release studies}

In vitro ZMT release profile of selected nanoparticles was determined using dialysis membrane in USP II dissolution apparatus (Yang et al., 1999). The nanoparticles were kept in dialysis membrane-150 with a molecular weight cut-off of range of 12 - $14 \mathrm{kDa}$ (Hi-Media, Mumbai, India) and tied with rotating paddle at $100 \mathrm{rpm}$. An aliquot was withdrawn at different time intervals of $0,5,10,20,30 \ldots$ up to $240 \mathrm{~min}$ and analyzed simultaneously by UV spectrophotometry at $222 \mathrm{~nm}$. Further drug release pattern was fit in different kinetic modeling (Costa and Lobo, 2001).

\section{Zeta potential measurement}

To study surface charge and stability, a zeta potential of optimized nanoparticles was measured using a Zetasize analyser (Nano partica SZ 100, Horriba, Kyoto, Japan). The analysis was based on laser doppler electrophoresis (LDE) method.
Table 1: Coded levels of independent variables and response variables.

\begin{tabular}{cccc}
\hline \multirow{2}{*}{ Independent Variables } & \multicolumn{3}{c}{ Levels } \\
\cline { 2 - 4 } & Low (-1) & Medium (0) & High (+1) \\
\hline $\mathrm{X}_{1}$ :Chitosan $(\mathrm{mg} / \mathrm{ml})$ & 1 & 2 & 3 \\
$\mathrm{X}_{2}$ :STPP $(\mathrm{mg} / \mathrm{ml})$ & 0.5 & 1 & 1.5 \\
Dependent Variables & \multicolumn{3}{|}{ Goal } \\
Y1- Particles Size (nm) & Minimum \\
Y $_{2}$ Entrapment Efficiency (\%) & \multicolumn{3}{c}{ Maximum } \\
\hline
\end{tabular}

Table 2: Design matrix of $3^{2}$ factorial design.

\begin{tabular}{ccccc}
\hline \multirow{2}{*}{ Run } & $\begin{array}{c}\text { Independent Variables with } \\
\text { coded levels }\end{array}$ & \multicolumn{2}{c}{$\begin{array}{c}\text { Response variables with } \\
\text { obtained results }\end{array}$} \\
\cline { 2 - 5 } & $\mathbf{X}_{\mathbf{1}}$ (CS) & $\mathbf{X}_{\mathbf{2}}$ (STPP) & $\mathbf{Y}_{\mathbf{1}}$ (PS)* & $\mathbf{Y}_{\mathbf{2}}$ (EE)* \\
\hline Z1 & -1 & -1 & $151 \pm 11$ & $78.1 \pm 4$ \\
Z2 & -1 & 0 & $158 \pm 10$ & $72.3 \pm 3$ \\
Z3 & -1 & +1 & $224 \pm 09$ & $68.8 \pm 2.5$ \\
Z4 & 0 & -1 & $155 \pm 15$ & $78.3 \pm 6$ \\
Z5 & 0 & 0 & $161 \pm 12$ & $80.6 \pm 2$ \\
Z6 & 0 & +1 & $310 \pm 13$ & $73.7 \pm 5$ \\
Z7 & +1 & -1 & $430 \pm 13$ & $81.2 \pm 2$ \\
Z8 & +1 & 0 & $680 \pm 15$ & $76.2 \pm 4$ \\
$Z 9$ & +1 & +1 & $880 \pm 18$ & $75.4 \pm 4.5$ \\
\hline${ }^{*} n=3$ & \multicolumn{5}{c}{}
\end{tabular}

Table 3: Fit summary model for the measured responses.

\begin{tabular}{ccccc}
\hline \multirow{2}{*}{ Source } & \multicolumn{2}{c}{$\mathbf{Y}_{\mathbf{1}}$ (PS) } & \multicolumn{2}{c}{$\mathbf{Y}_{\mathbf{2}}$ (EE) } \\
\cline { 2 - 5 } & F value & P value & F value & P value \\
\hline Linear versus mean & 10.33 & 0.0114 & 6.25 & 0.0341 \\
2FI versus linear & 1.27 & 0.3116 & 0.33 & 0.5903 \\
Quadratic versus 2FI & 14.85 & 0.0278 & 12.79 & 0.0340 \\
\hline
\end{tabular}

2FI= Two factor interaction model

Table 4: Model summary statistics of response to select suitable model to fit data.

\begin{tabular}{ccccccc}
\hline \multirow{2}{*}{ Source } & \multicolumn{3}{c}{ Y $_{\mathbf{1}}$ (PS) } & \multicolumn{3}{c}{ Y $_{2}$ (EE) } \\
& Adj R $^{2}$ & Pred. R & PRESS & Adj R & Pred. R & PRESS \\
\hline Linear & 0.6998 & 0.5081 & 238500 & 0.5676 & 0.3762 & 95.02 \\
2FI & 0.7126 & 0.4088 & 286600 & 0.5133 & 0.2377 & 111.14 \\
Quadratic & 0.9560 & 0.8337 & 80596.7 & 0.9149 & 0.7175 & 43.04 \\
\hline
\end{tabular}

Adj $R^{2}$ : adjusted determination coefficient; Pred. $R^{2}$ : predicted determination coefficient, PRESS: predicted residual sum of square.

Table 5: ANOVA for measured responses.

\begin{tabular}{|c|c|c|c|c|}
\hline \multirow{2}{*}{ Source } & \multicolumn{2}{|c|}{$\mathrm{Y}_{1}$ (PS) } & \multicolumn{2}{|c|}{$\mathrm{Y}_{2}(\mathrm{EE})$} \\
\hline & F value & $P$ value & F value & $P$ value \\
\hline $\begin{array}{c}\text { Significant } \\
\text { model }\end{array}$ & \multicolumn{2}{|c|}{ Quadratic Model } & \multicolumn{2}{|c|}{ Quadratic Model } \\
\hline Model & 35.797 & 0.0071 & 18.191 & 0.0188 \\
\hline $\mathrm{X}_{1}$ & 118.64 & 0.0017 & 19.012 & 0.0223 \\
\hline $\mathrm{X}_{2}$ & 22.375 & 0.0179 & 44.472 & 0.0069 \\
\hline $\mathrm{X}_{1} \mathrm{X}_{2}$ & 8.2788 & 0.0637 & 1.888 & 0.2630 \\
\hline $\mathrm{X}_{1}{ }^{2}$ & 29.584 & 0.0122 & 24.978 & 0.0154 \\
\hline $\mathrm{X}_{2}{ }^{2}$ & 0.1081 & 0.7639 & 0.604 & 0.4936 \\
\hline C.V \% & \multicolumn{2}{|c|}{15.14} & \multicolumn{2}{|c|}{1.66} \\
\hline $\begin{array}{l}\text { Adequate } \\
\text { Precision }\end{array}$ & \multicolumn{2}{|c|}{16.649} & \multicolumn{2}{|c|}{14.019} \\
\hline
\end{tabular}

C.V: Coefficient of variation 
Surface Morphology: Transmission Electron Microscopy (TEM)

Morphological examination of optimized nanoparticles was performed using a transmission electron microscope (Philips-CM 200, Eindhoven, Netherland). The samples were immobilized in the carbon coated copper grids, dried and then examined without being stained.

\section{Statistical Analysis}

The design and statistical analysis were performed by licensed version of Design-Expert ${ }^{\circledR}$ V8 (DX8) Software (Stat ease MN, USA). The measured responses for particle size and entrapment efficiency were subjected to the regression analysis, polynomial model fitting, analysis of variance (ANOVA) and other statistical parameters.

$Y=\beta_{0}+\beta_{1} X_{1}+\beta_{2} X_{2}+\beta_{3} X_{1} X_{2}+\beta_{4} X_{1}^{2}+\beta_{5} X_{2}^{2}$

The generalized regression model equation is represented in Equation 2, where $Y$ is a response variable (CTQ), $\beta_{0}$ is the arithmetic mean response of all 9 runs; and $\beta_{1}-\beta_{5}$ represent estimated coefficients for the factors $X_{1}$ and $X_{2}$, respectively. The main effects $\left(X_{1}\right.$ and $\left.X_{2}\right)$ represent the average result whereas the term $X_{1} X_{2}$ represent the interaction, shows how the response changes when 2 factors are simultaneously changed. The second order terms $\mathrm{X}_{1}{ }^{2}$ and $\mathrm{X}_{2}{ }^{2}$ indicate the quadratic effect (Mandlik et al., 2009).

\section{Numerical and graphical optimization and validation}

To confirm the applicability of the established model equations for optimization of responses, a design was validated using numerical and graphical optimization method (Statease-1, 2012). The numerical optimization was applied to target the goal for each response to generate the optimal conditions. In graphical optimization, minimum and maximum values for each response were set to create an overlay plot highlighting operating window (area of operability). As per the predicted values of independent variables, nanoparticles were prepared and characterized later differences in the predicted and experimentally obtained values of responses were compared by using student's $t$ test.

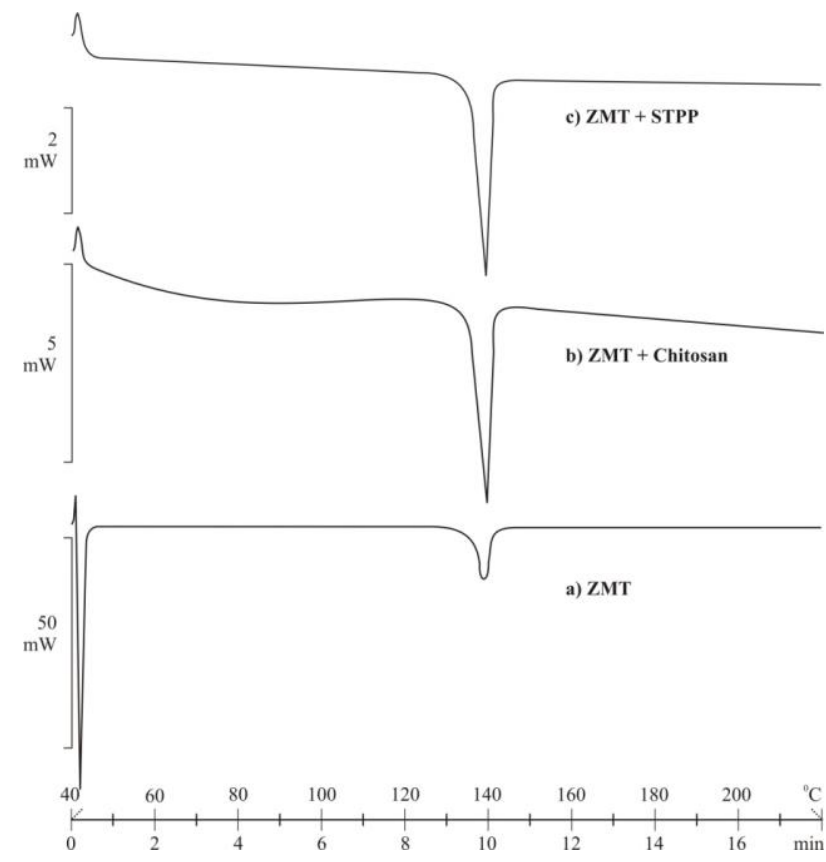

Fig. 1. DSC Thermograms of a) ZMT, b) ZMT+ Chitosan and c) ZMT+STPP.

\section{RESULTS AND DISCUSSION}

The present study was researched to prepare nanoparticles of an antimigraine drug, ZMT, in combination with the chitosan biodegradable polymer using the ionic interaction method.

Drug-excipients interaction studies were performed by differential scanning colorimetry and FTIR spectroscopy. The DSC thermogram of pure ZMT and physical mixture of ZMT with both chitosan and STPP exhibited sharp endothermic peak at $140^{\circ} \mathrm{C}$ corresponding to melting point of pure drug as shown in the Fig. 1. The FT-IR spectra

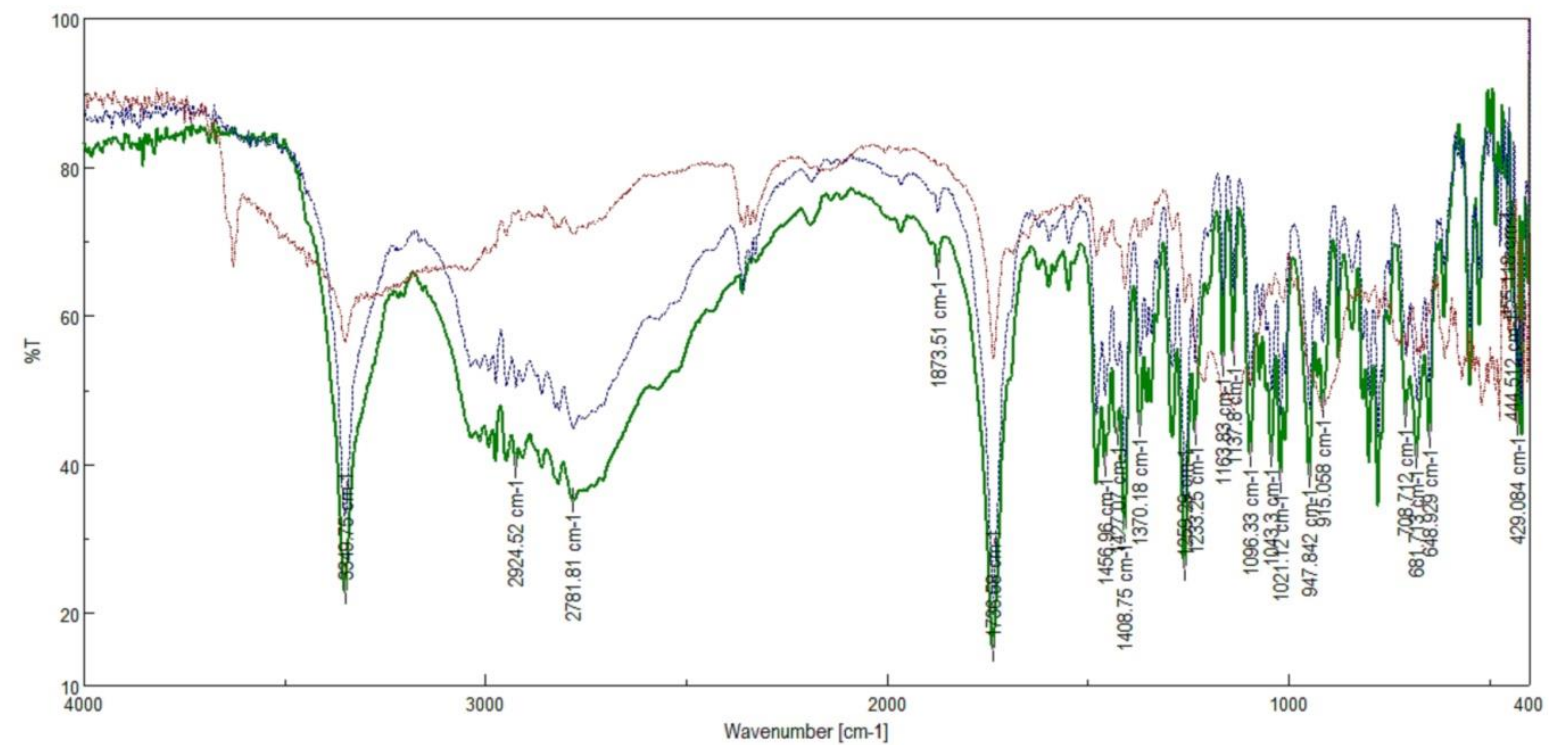

Fig. 2. FTIR Spectra of pure a) ZMT, b) ZMT+ chitosan and c) ZMT+ STPP. 


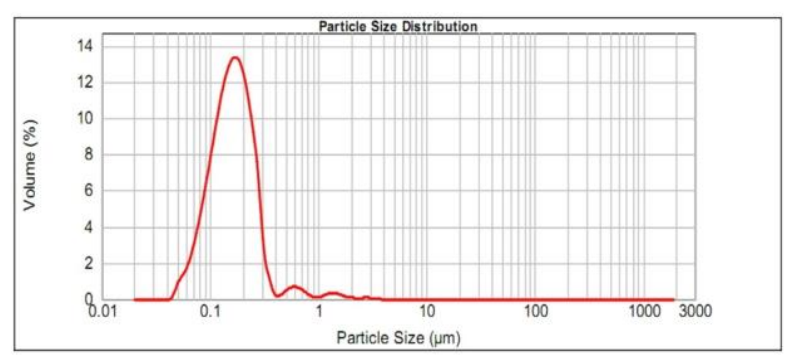

Fig. 3. Particle size distribution of $Z 5$ batch [d $(0.5)=161 \mathrm{~nm}]$.

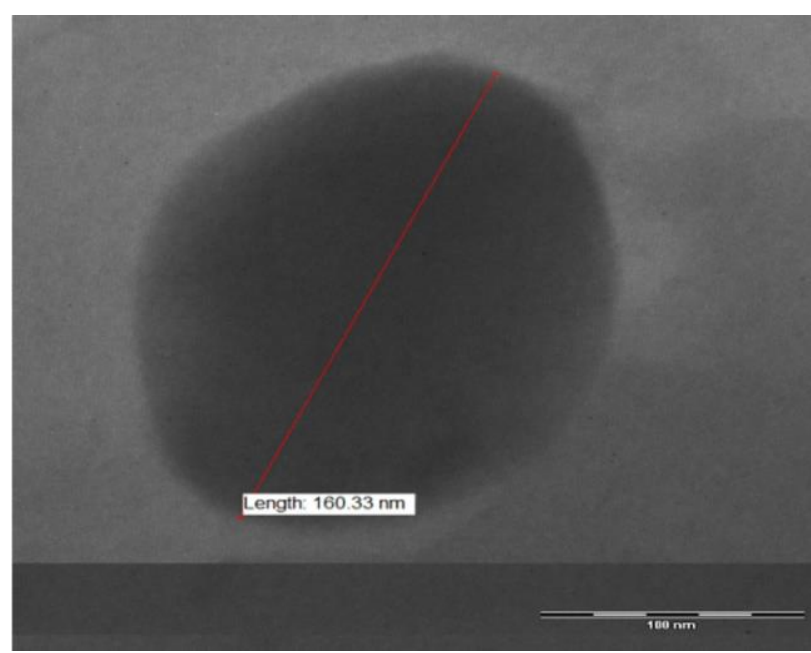

Fig. 4. TEM micrograph of $Z 5$ nanoparticles.

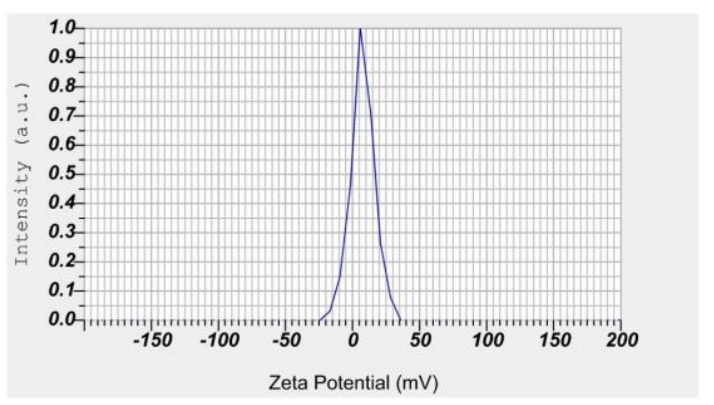

Fig. 5. Zeta potential of $\mathrm{Z} 5$ nanoparticles $(+23.7 \mathrm{mV})$.

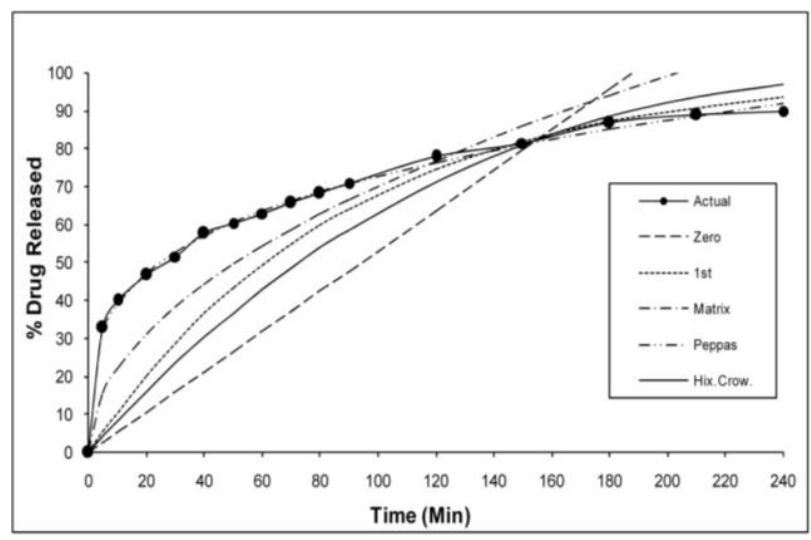

Fig. 6. Drug release profile and model fitting for optimized Z5 nanoparticles. of pure ZMT showed characteristics peaks at $1234 \mathrm{~cm}^{-1}$ indicates $\mathrm{C}-\mathrm{O}$ bond, $1409 \mathrm{~cm}^{-1}$ indicates $\mathrm{C}=\mathrm{C}$ stretching vibration, $1733 \mathrm{~cm}^{-1}$ indicates $\mathrm{C}=\mathrm{O}$ stretching vibration and the peak at $3350 \mathrm{~cm}^{-1}$ indicates $\mathrm{N}-\mathrm{H}$ bond which is depicted in overlain spectra (Fig. 2). FT-IR studies showed the formation of a hydrogen bond between drug and chitosan. Similarly, IR spectra of pure ZMT and its physical mixtures (1:1) did not show any significant differences which evidenced the compatibility of ZMT and excipients used.

The preliminary studies were carried out for the selection of new approach for ionic interaction using a cyclomixer, concentration range for chitosan and STPP. The cationic nature of chitosan allows ionic cross-linking with multivalent anions of STPP to form nanoparticles. As per $3^{2}$ factorial design, nine batches of ZMT-loaded nanoparticles were prepared by changing the concentration of two independent variables, chitosan and STPP. The design matrix of nine runs with obtained results is mentioned in Table 2.

The values of all the 9 batches showed variation in particle size from $151 \mathrm{~nm}$ to $800 \mathrm{~nm}$ and $68.8 \%$ to $81.2 \%$ in entrapment efficiency. The particle size increases dramatically with increasing concentration of both chitosan and STPP but amongst them, an effect of chitosan concentration was significant than STPP. Initially EE tended to increase with increase in concentration of ZMT however maximum drug entrapment was obtained for Z7 batch prepared with highest concentrations of chitosan and lowest concentration of STPP but the particle size was $430 \mathrm{~nm}$ which was beyond the desired goal.

The entrapment efficiency obtained for a Z5 batch was $80.6 \%$ with the desired particle size $(161 \mathrm{~nm})$ so it was considered as an optimized batch for further characterization. The particle size distribution, TEM micrograph of optimized Z5 nanoparticles is presented in Fig. 3 and 4 respectively. The TEM micrograph showed spherical shaped nanoparticles with smooth surfaces. The magnitude of the zeta potential predicts colloidal stability. Zeta potential of the optimized Z5 nanoparticles was found to be $+23.7 \mathrm{mV}$ (Fig. 5) which assures the stability of colloidal nanoparticles. The positive values of zeta potential specify that the nanoparticles surface was positively charged which may be attributed due to presence of amino groups of chitosan on the surface of nanoparticles (Thode et al., 2000; Vila et al., 2004). Zeta Potential values greater than $+20 \mathrm{mV}$ or less than $-20 \mathrm{mV}$ typically have high degrees of stability. A high zeta potential value of nanoparticles shows the extent of electrostatic repulsion between all the adjacent like charged particles which contributes to the prevention of colloidal aggregates (Gan et al., 2005).

The in vitro release profile of optimized $\mathrm{Z} 5$ nanoparticles in phosphate buffer solution ( $\mathrm{pH} 7.4)$ is shown in Fig. 6. Initial burst release was observed, with about $40 \%$ ZMT being released in $10 \mathrm{~min}$ which may be due to availability of ZMT on the surface of nanoparticles (Seju et al., 2011), followed by slow release of drug which was encapsulated in nanoparticles. More than $90 \%$ drug was released at $4^{\text {th }} \mathrm{h}$. The slow release was due to slow diffusion rate of ZMT through polymeric nanoparticles (Shah et al., 2009). The data obtained from in vitro drug release studies was fitted to different dissolution kinetics models and KorsmeyerPeppas model was found to be a best fit model with $\mathrm{R}^{2}$ 0.998 . The release exponent $(n)$ value was $0.266(<0.5)$ which characterizes the Fickian diffusion transport mechanism (Peppas, 1985).

The obtained data were fitted into different polynomial mathematical model and a best fit model was 


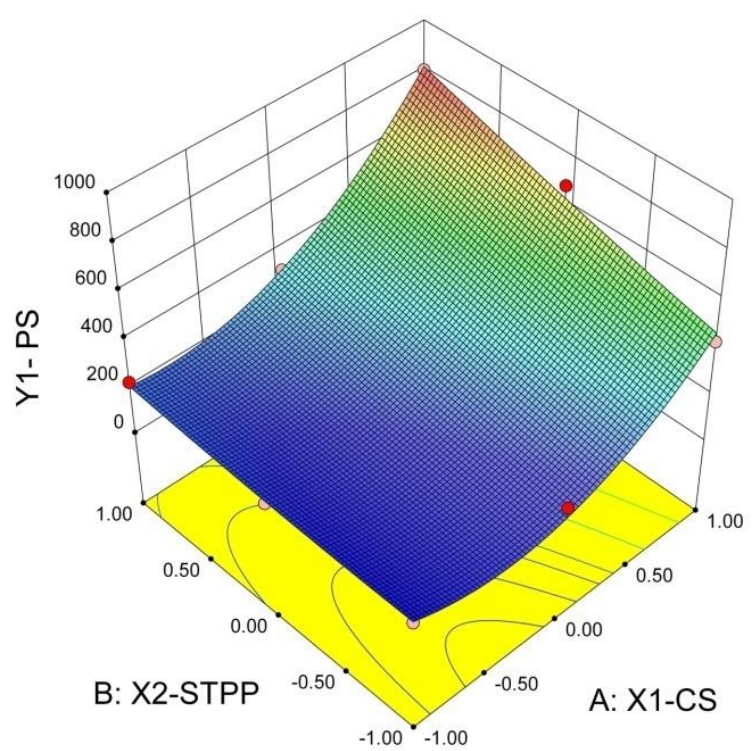

Fig. 7. Response surface plot and contour plot for $Y_{1}$ (PS).

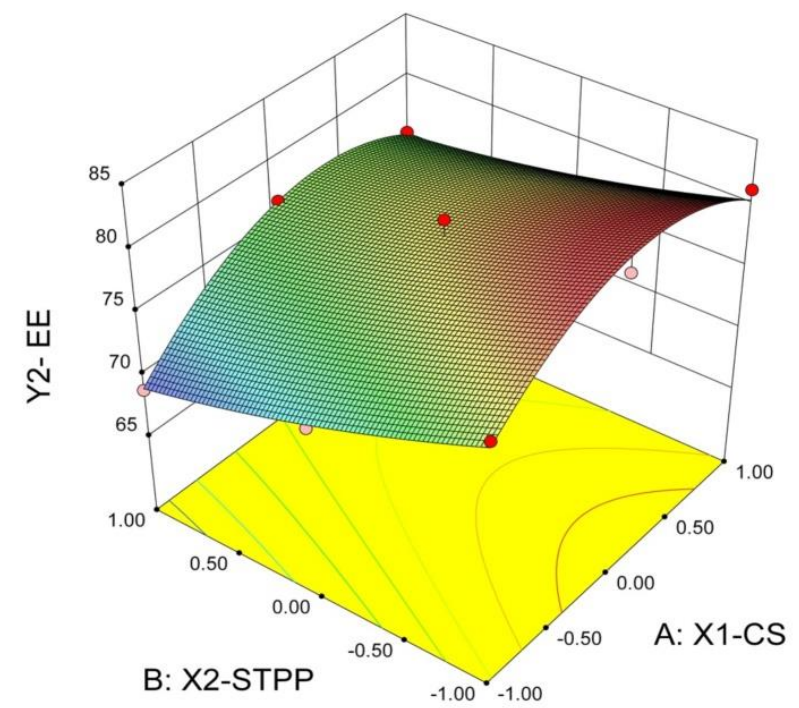

Fig. 8. Response surface plot and contour plot for $Y_{2}$ (EE).

suggested by the design expert software depending on parameters such as $p$ value, adjusted determination coefficient (adj. $\mathrm{R}^{2}$ ), predicted determination coefficient (pred. $\mathrm{R}^{2}$ ) and PRESS (predicted residual sum of square) values as mentioned in Table 3 and Table 4 . The predicted $R^{2}$ value for $Y_{1}$ and $Y_{2}$ are in reasonable agreement with the adjusted $\mathrm{R}^{2}$ value. The analysis of variance (ANOVA) for both responses shown in Table 5 was used to measure the significance of models. For the particle size $\left(\mathrm{Y}_{1}\right)$, both linear and quadratic model were found to be fit with respect to its $p$ value (Table 3). The adjusted $\mathrm{R}^{2}$ and PRESS values were compared and lesser the PRESS value, better is the model

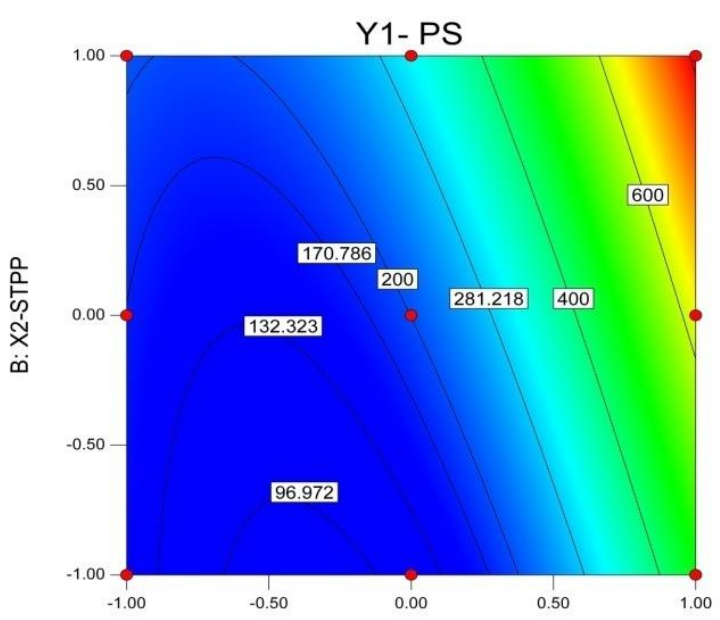

A: $\mathrm{X} 1-\mathrm{CS}$

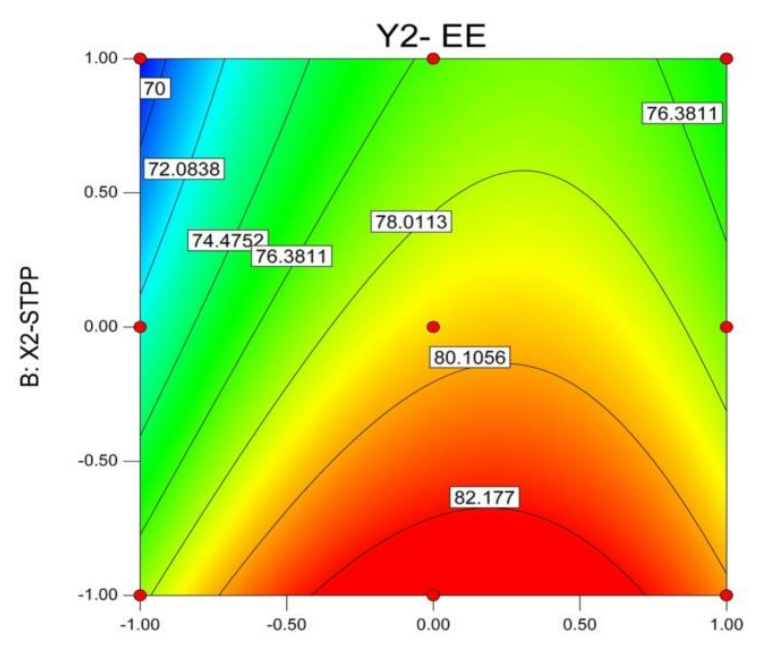

A: $\mathrm{X} 1-\mathrm{CS}$

fitting. The adjusted $\mathrm{R}^{2}$ value and PRESS value for the quadratic model were found to be 0.9560 and 80596.7 respectively, indicating adequate fitting of model.

The model F-value of 35.797 implies the significant model. A $p$ value of "Prob > F" was 0.0071 less than 0.0500 indicate model terms are significant. Hence, a quadratic model was found be significant for particle size (Bolton and Bon, 2004). Adequate precision is a measure of the range in predicted response relative to its associated error, it measures signal to noise ratio. The ratio for $\mathrm{Y}_{1}$ was 16.649 indicates an adequate signal. (A desired value should be 4 or more.). The 
best fit quadratic model equation generated for particles size $\left(\mathrm{Y}_{1}\right)$ is shown in equation 3 ,

$Y_{1}(P S)=$

200.67+229.50 $X_{1}+99.67 X_{2}+74.25 X_{1} X_{2}+198.50 X_{1}^{2}+12.00 X_{2}^{2}--$ (3)

In equation 3 , the average coefficient of quadratic model was 200.67. The coefficient for individual chitosan and STPP were 229.50 and 99.67 respectively. This indicates that effect of chitosan concentration on nanoparticle size was more pronounced than STPP. This was also confirmed graphically by $3 \mathrm{D}$ response surface plot and 2D contour plot. The elliptical contour lines signify a best quadratic effect (Fig. 7).

For entrapment efficiency, the quadratic model was found to be fit with respect to its $p$ value as shown in Table 3 . The adjusted $R^{2}$ and PRESS values were compared (Statease-2, 2012; Kumar and Sawant, 2013). The adjusted $R^{2}$ value and PRESS value for the quadratic model were found to be 0.9149 and 43.04 respectively, indicating adequate fitting of model. The quadratic model was found significant for entrapment efficiency. The model F-value of $18.191 \mathrm{im}$ plies the model is significant. A $p$ value of "Prob $>$ F" was 0.0188 less than 0.0500 indicate model terms are significant. The signal to noise ratio for $Y_{2}$ was 14.019 indicates an adequate signal. The best fit quadratic model equation generated for entrapment efficiency $\left(\mathrm{Y}_{2}\right)$ is shown in equation 4 ,

$Y_{2}(E E)=79.36+2.26 X_{1}+3.46 X_{2}-0.87 X_{1} X_{2}-4.50 X_{1}^{2}+0.70 X_{2}^{2}---(4)$

The equation 4 reveals that both the factors (chitosan and STPP) have positive effect on entrapment of ZMT in nanoparticles but their interaction was found to be slightly negative. The $3 \mathrm{D}$ response surface plots and $2 \mathrm{D}$ contour plots are shown in Fig. 8. The elliptical contour lines confirm the significant quadratic effect of independent variables on EE.

It was observed from the response surface plots and contour plots that optimum concentration of chitosan (2 $\mathrm{mg} / \mathrm{ml})$ and STPP $(1 \mathrm{mg} / \mathrm{ml})$ were required for preparation of nanoparticles with desired particle size and entrapment efficiency. From the results, the optimum levels of independent variables were screened out by regression analysis. Through numerical and graphical optimization process, the goals of responses were set to $175 \mathrm{~nm}$ for particle size and $80 \%$ for entrapment efficiency. Further to validate the predicted responses, software suggested levels -0.03 and 0.20 for $X_{1}$ and $X_{2}$ respectively. The calculated desirability factor for suggested levels was 1, which indicated suitability of the model. After graphical optimization, overlay plot (Fig. 9) displays the area of feasible response values in the factor space. The yellow highlighted area shows desirability of 1 suggesting optimized zone of operability. Further prepared nanoparticles did not show significant difference between predicted values and experimentally obtained values ( $Y_{1} .178 .00 \mathrm{~nm}$ and $\left.\mathrm{Y}_{2} .80 .55 \%\right)$. The $t$-test was applied between the actual and predicted values of $X_{1}$ and $X_{2}$ and $p$ value $>0.05$ was observed. Therefore, it confirms the validity of quadratic model equations to achieve desired responses of nanoparticles.

\section{CONCLUSION}

The present study demonstrated the use of two factor and three level factorial design as an experimental design method to understand the effect of various input variables in the prediction of desired responses with limited number of experiments. IR spectroscopy and DSC studies confirmed non-interaction between drug and excipients. ZMT loaded chitosan nanoparticles were prepared by modified

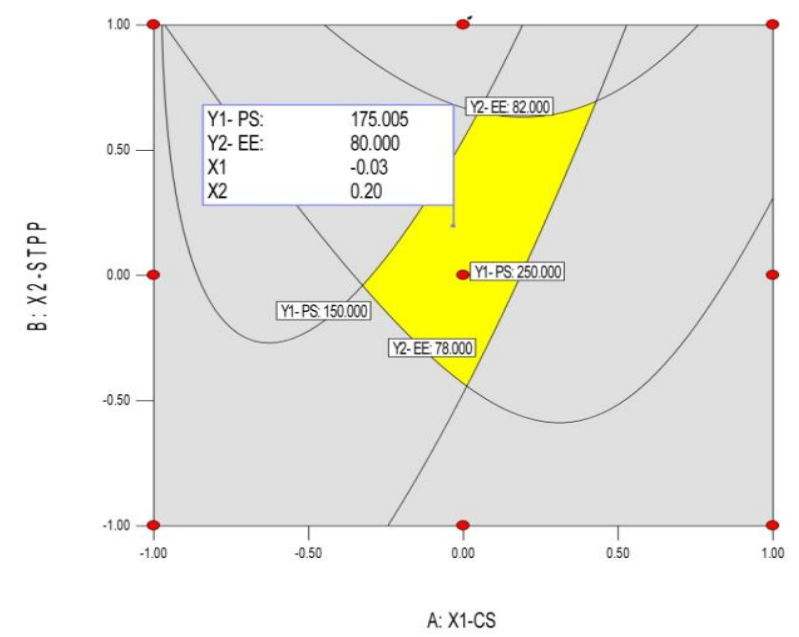

Fig. 9. Graphical optimization-overlay plot of $Y_{1}$ and $Y_{2}$ (Design Space).

ionic gelation method. The optimized NPs had small PS and high entrapment of ZMT. These stable nanoparticles exhibited initial burst followed by slow release and followed Fickian diffusion type release kinetics. Both responses were statistically analyzed with significant results. Quadratic polynomial equation was validated using numerical and graphical optimization. Thus, using systematic factorial design approach, desirable goals can be achieved in shortest possible time with reduced number of experiments.

\section{ACKNOWLEDGEMENT}

The authors are grateful to Cipla Ltd, Mumbai, India, for providing a gift sample of ZMT.

\section{REFERENCES}

Agnihotri, S. A., Mallikarjuna, N. N., \& Aminabhavi, T. M. (2004). Recent advances on chitosan-based micro- and nanoparticles in drug delivery. Journal of Controlled Release, 100(1), 5-28. [DOI]

Amidi, M., Mastrobattista, E. Jiskoot, W. \& Hennink, W. E. (2010). Chitosan-based delivery systems for protein therapeutics and antigens. Advanced Drug Delivery Reviews, 62(1), 59-82. [DOI]

Antony, J. (2014). Fundamentals of DOE. Design of experiments for engineers and scientists (2nd ed., pp. 7-17). Elsevier. [DOI]

Charlesworth, B., \& Dowson, A. J. (2002). Review of zolmitriptan and its clinical applications in migraine. Expert Opinion on Pharmacotherapy, 3(7), 993-1005. [DOI]

Christoper, G. P., Raghavan, C. V., Siddharth, K., Kumar, M. S., \& Prasad, R. H. (2014). Formulation and optimization of coated PLGA - Zidovudine nanoparticles using factorial design and in vitro in vivo evaluations to determine brain targeting efficiency. Saudi Pharmaceutical Journal, 22(2), 133-140. [DOI]

Costa, P., \& Lobo, J. M. S. (2001). Modeling and comparison of dissolution profiles. European journal of pharmaceutical sciences, 13(2), 123-133. [DOI]

Dixon, R., Warrander, A. (1997). The clinical pharmacokinetics of Zolmitriptan. Cephalalgia, 17(18), 15-20.

Emami, J., Boushehri, M. S., \& Varshosaz, J. (2014). Preparation, characterization and optimization of glipizide controlled release nanoparticles. Research in pharmaceutical sciences, 9(5), 301-314.

Gan, Q., Wang, T., Cochrane, C., \& McCarron, P. (2005). Modulation of surface charge, particle size and morphological properties of chitosanTPP nanoparticles intended for gene delivery. Colloids and Surfaces B: Biointerfaces, 44(2), 65-73. [DOI] 
Goadsby, P. J., \& Yates, R. (2006). Zolmitriptan Intranasal: A Review of the Pharmacokinetics and Clinical Efficacy. Headache: The Journal of Head and Face Pain, 46(1), 138-149. [DOI]

Koukaras, E. N., Papadimitriou, S. A., Bikiaris, D. N., \& Froudakis, G. E. (2012). Insight on the formation of chitosan nanoparticles through ionotropic gelation with tripolyphosphate. Molecular pharmaceutics, 9(10), 2856-2862. [DOI]

Kumar, A., \& Sawant, K. (2013). Encapsulation of exemestane in polycaprolactone nanoparticles: optimization, characterization, and release kinetics. Cancer nanotechnology, 4(4-5), 57-71. [DOI]

Lewis, G. A., Mathieu, D., \& Phan-Tan-Luu, R. (1998). Factor Influence studies- Applications of Full and Fractional Factorial Designs. Pharmaceutical experimental design. (1st ed., pp 78-100). CRC Press. [DOI]

Mandlik, S. K., Adhikari, S., \& Ranpise, N. S. (2013). Formulation and invitro characterization of chitosan biodegradable nanoparticles of zolmitriptan for migraine treatment. Pharmacie Globale, International journal of comprehensive Pharmacy, 4(1), 1-5.

Mandlik, S. K., Nandare, D. S., Joshi, M. M., Chudiwal, P. D., \& Jain, K. S. (2009). Statistical Optimization of Orodispersible Tablets Containing Telmisartan Using Factorial Design and Response Surface Methodology. Research Journal of Pharmacy and Technology, 2(3), 548-551.

Mehta, A. K., Yadav, K. S., \& Sawant, K. K. (2007). Nimodipine loaded PLGA nanoparticles: formulation optimization using factorial design, characterization and in vitro evaluation. Current drug delivery, 4(3), 185193. [DOI]

Mincea, M., Negrulescu, A., \& Ostafe, V. (2012). Preparation, modification, and applications of chitin nanowhiskers: a review. Reviews on Advanced Materials Science 30(3), 225-242.

Neves, A. L., Milioli, C. C., Müller, L., Riella, H. G., Kuhnen, N. C., \& Stulzer, H. K. (2014). Factorial design as tool in chitosan nanoparticles development by ionic gelation technique. Colloids and Surfaces A: Physicochemical and Engineering Aspects, 445, 34-39. [DOI]

Parhi, R., Suresh, P., \& Patnaik, S. (2015). Formulation optimization of PVA/HPMC cryogel of Diltiazem $\mathrm{HCl}$ using 3-level factorial design and evaluation for ex vivo permeation. Journal of Pharmaceutical Investigation, 45(3), 319-327. [DOI]
Peppas, N. A. (1985). Analysis of Fickian and non-Fickian drug release from polymers. Pharmaceutica Acta Helvetiae, 60(4), 110-111.

Seju, U., Kumar, A., \& Sawant, K. K. (2011). Development and evaluation of olanzapine-loaded PLGA nanoparticles for nose-to-brain delivery: in vitro and in vivo studies. Acta biomaterialia, 7(12), 4169-4176.

Shah, N., Chaudhari, K, Dantuluri, P., Murthy, R. S. R., \& Das, S. (2009). Paclitaxel-loaded PLGA nanoparticles surface modified with transferrin and Pluronic ${ }^{\circledR}$ P85, an in vitro cell line and in vivo biodistribution studies on rat model. Journal of drug targeting, 17(7), 533-542. [DOI]

Thode, K., Müller, R. H., \& Kresse, M. (2000). Two-time window and multiangle photon correlation spectroscopy size and zeta potential analysis - Highly sensitive rapid assay for dispersion stability. Journal of pharmaceutical sciences, 89(10), 1317-1324. [DOI]

Tiyaboonchai, W. (2003). Chitosan nanoparticles: a promising system for drug delivery. Naresuan University Journal, 11(3), 51-66.

Uemura, N., Onishi, T., Mitaniyama, A., Kaneko, T., Ninomiya, K., Nakamura, K., \& Tateno, M. (2005). Bioequivalence and Rapid Absorption of Zolmitriptan Nasal Spray Compared with Oral Tablets in Healthy Japanese Subjects. Clinical Drug Investigation, 25(3), 199-208. [DOI]

Vila, A., Sánchez, A., Janes, K., Behrens, I., Kissel, T., Jato, J. L. V., \& Alonso, M. J. (2004). Low molecular weight chitosan nanoparticles as new carriers for nasal vaccine delivery in mice. European Journal of Pharmaceutics and Biopharmaceutics, 57(1), 123-131. [DOI]

Wang, X., Chi, N., \& Tang, X. (2008). Preparation of estradiol chitosan nanoparticles for improving nasal absorption and brain targeting. European journal of pharmaceutics and biopharmaceutics, 70(3), 735-740. [DOI]

Yang, S. C., Lu, L. F., Cai, Y., Zhu, J. B., Liang, B. W., \& Yang, C. Z. (1999) Body distribution in mice of intravenously injected camptothecin solid lipid nanoparticles and targeting effect on brain. Journal of controlled release, 59(3), 299-307. [DOI]

Zhang, L., \& Mao, S. (2016). Applications of quality by design (QbD) and its tools in drug delivery. Asian Journal of Pharmaceutical Sciences, 11(1), 144-145. [DOI] 